\title{
Revascularization of ischemic tissues by PDGF-CC via effects on endothelial cells and their progenitors
}

\author{
Xuri Li, ${ }^{1,2}$ Marc Tjwa, ${ }^{1}$ Lieve Moons, ${ }^{1}$ Pierre Fons, ${ }^{3}$ Agnes Noel, ${ }^{4}$ Annelii Ny, ${ }^{1}$ Jian Min Zhou, ${ }^{2}$ \\ Johan Lennartsson, ${ }^{5}$ Hong Li, ${ }^{2}$ Aernout Luttun, ${ }^{1}$ Annica Pontén, ${ }^{2}$ Laetitia Devy, ${ }^{4}$ Ann Bouché, ${ }^{1}$ \\ Hideyasu Oh, ${ }^{1}$ Ann Manderveld,, ${ }^{1}$ Silvia Blacher, ${ }^{4}$ David Communi, 6 Pierre Savi, ${ }^{3}$ Françoise Bono, ${ }^{3}$ \\ Mieke Dewerchin, ${ }^{1}$ Jean-Michel Foidart, ${ }^{4}$ Monica Autiero, ${ }^{1}$ Jean-Marc Herbert, ${ }^{3}$ Désiré Collen, ${ }^{1}$ \\ Carl-Henrik Heldin, ${ }^{5}$ Ulf Eriksson, ${ }^{2}$ and Peter Carmeliet ${ }^{1}$
}

${ }^{1}$ The Center for Transgene Technology and Gene Therapy, Flanders Interuniversitary Institute for Biotechnology (VIB), University of Leuven, Leuven, Belgium. ${ }^{2}$ Ludwig Institute for Cancer Research, Karolinska Institute, Stockholm, Sweden. ${ }^{3}$ Cardiovascular/Thrombosis Research Department, Sanofi-Aventis, Toulouse, France. ${ }^{4}$ Laboratoire de Biologie des Tumeurs et du Développement, Université de Liège, Liège, Belgium. ${ }^{5}$ Ludwig Institute for Cancer Research, Uppsala, Sweden. ${ }^{6}$ Institut de Recherche Interdisciplinaire en Biologie Humaine et Moléculaire (IRIBHM), Free University of Brussels, Brussels, Belgium.

\begin{abstract}
The angiogenic mechanism and therapeutic potential of PDGF-CC, a recently discovered member of the VEGF/ PDGF superfamily, remain incompletely characterized. Here we report that PDGF-CC mobilized endothelial progenitor cells in ischemic conditions; induced differentiation of bone marrow cells into ECs; and stimulated migration of ECs. Furthermore, PDGF-CC induced the differentiation of bone marrow cells into smooth muscle cells and stimulated their growth during vessel sprouting. Moreover, delivery of PDGF-CC enhanced postischemic revascularization of the heart and limb. Modulating the activity of PDGF-CC may provide novel opportunities for treating ischemic diseases.
\end{abstract}

\section{Introduction}

The PDGF family consists of 4 members: PDGF-AA, PDGF-BB, and the newly discovered PDGF-CC and PDGF-DD (1-3). Homoand heterodimers of these ligands bind, with distinct selectivity, dimeric complexes of the receptor tyrosine kinases PDGF$\mathrm{R} \alpha$ and PDGF-R $\beta$ (4). PDGF-AA selectively binds PDGF-R $\alpha \alpha$; PDGF-BB binds PDGF-R $\alpha \alpha,-R \beta \beta$, and $-\mathrm{R} \alpha \beta$; while PDGF-CC binds PDGF-R $\alpha \alpha$ and $-R \alpha \beta(1,5)$. In the vessel wall, PDGF-R $\alpha$ and $-R \beta$ are expressed on mural cells, i.e. pericytes and smooth muscle cells (SMCs), and activation by PDGF-AA or -BB stimulates the growth and migration of these cells (6-12). By recruiting PDGF-R $\beta^{+}$ mural cells, PDGF-BB, secreted by ECs, plays an essential role in the maturation of nascent blood vessels and tissue perfusion (7-13). PDGF-BB also promotes microvessel growth indirectly by stimulating mural cells to produce VEGF $(12,14)$. As PDGF-AA also affects SMCs (6), it might affect angiogenesis indirectly. When administered alone, PDGF-BB destabilizes vessels by reducing EC survival as a result of impaired mural cell coverage (15), but, when coadministered with VEGF or FGF-2, PDGF-BB stimulates revascularization of the ischemic hind limb by promoting vessel growth and maturation (16-18). Conversely, inhibition of PDGF-R $\alpha$ and $-\mathrm{R} \beta$ signaling blocks angiogenesis in tumors and the ischemic retina, in part by inducing pericyte detachment (19-23).

Nonstandard abbreviations used: BAEC, bovine aortic EC; DiI-ac-LDL, 1,1'-dioctadecyl-3,3,3',3'-tetramethylindocarbocyanine perchlorate-acetylated LDL; EPC, endothelial progenitor cell; HMVEC, human microvascular EC; HUVEC, human umbilical venous EC; PAEC, porcine aortic EC; PAEC/R $\alpha$, PAECs stably overexpressing PDGF-R $\alpha$; PECAM, platelet endothelial cell adhesion molecule; pTyr, phosphotyrosine; SMC, smooth muscle cell; TM, thrombomodulin; VE-cadherin, vascular endothelial cadherin; WGA, wheat germ agglutinin.

Conflict of interest: The authors have declared that no conflict of interest exists.

Citation for this article: J. Clin. Invest. 115:118-127 (2005)

doi:10.1172/JCI200519189.
The role of PDGF-R $\alpha$ and $-\mathrm{R} \beta$ in regulating ECs remains more enigmatic. In vitro, PDGF-BB stimulated the growth and migration of particular ECs only (24-28), while PDGF-AA failed to affect ECs (24, 26-28), even despite PDGF-R $\alpha$ activation (25). This may relate to the fact that PDGF-R $\alpha$ and $-R \beta$ are present only on some, but not on all, ECs $(21,22,25-27,29-34)$. In addition, ECs in sprouting vessels express elevated levels of PDGF-R $\alpha$ and $-R \beta(22,25,33,34)$, while PDGF-R expression is downregulated in culture (25) or in quiescent vessels in healthy tissues (22). In vivo, both PDGF-AA and -BB stimulated microvessel growth in the cornea and chorionic allantoic membrane (35-37), but this angiogenic effect might be due to an indirect effect on mural cells. To complicate matters even more, another study reported that activation of PDGF-R $\alpha$ by PDGF-AA or -BB inhibited FGF-2-induced vessel growth (38).

The angiogenic role of PDGF-CC has been studied only to a limited extent. In the avascular mouse cornea, PDGF-CC stimulated microvessel growth, comparably to PDGF-BB but more potently than PDGF-AA, possibly because PDGF-CC activates PDGF-R $\alpha \alpha$ and $-R \alpha \beta$ (35). PDGF-CC also induced branching of preexisting vessels in the developing embryo (35) but failed to affect EC outgrowth from the aorta (5). PDGF-CC stimulates SMC growth $(1,5,39)$ and induces the release of VEGF (40), which suggests that this growth factor induced angiogenesis indirectly by affecting mural cells. However, it remains unknown whether PDGF-CC is capable of affecting ECs directly. Moreover, it is also unknown whether PDGF-CC affects the mobilization or differentiation of endothelial progenitors to sites of active vessel growth in the adult (a process termed "adult vasculogenesis"). In light of the fact that delivery of PDGF-BB alone is not capable of stimulating ischemic limb revascularization, it also remains to be determined whether single administration of PDGF-CC has any therapeutic potential to stimulate revascularization of the 

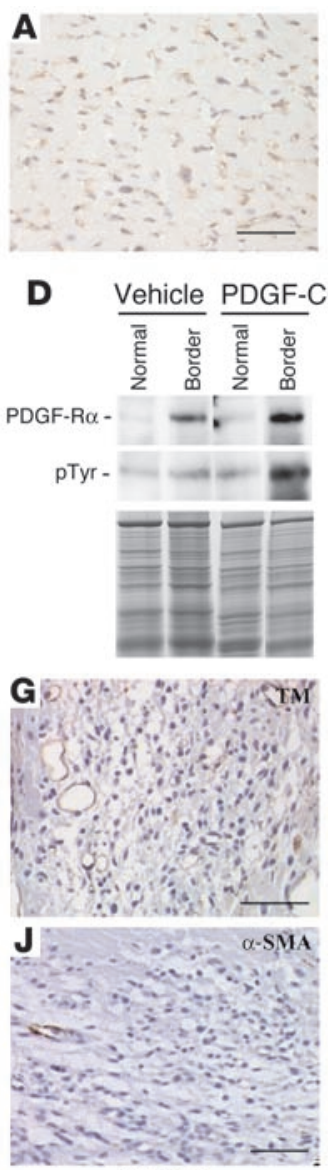

Vehicle
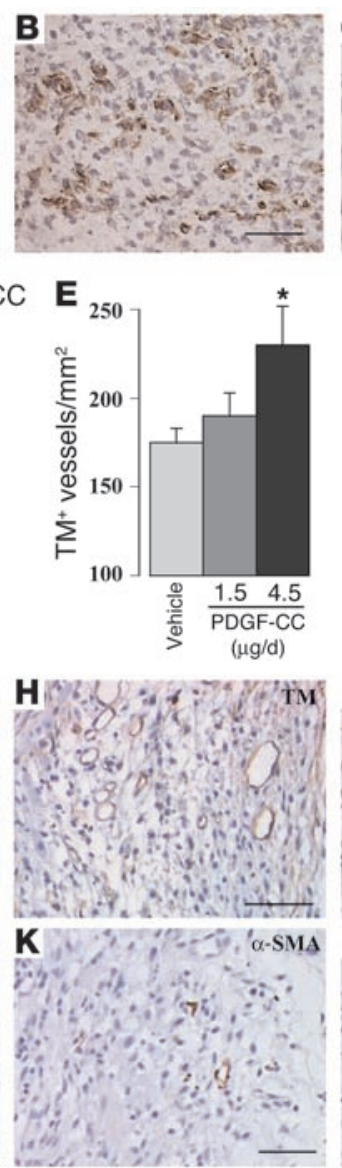

$1.5 \mu \mathrm{g}$ PDGF-CC/d

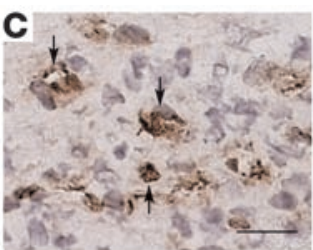

F
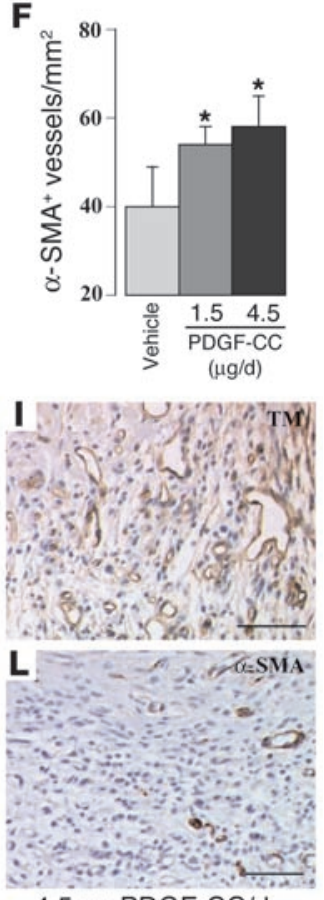

$4.5 \mu \mathrm{g}$ PDGF-CC/d

\section{Figure 1}

Therapeutic revascularization with PDGF-CC in ischemic heart. (A-C) Immunostaining, revealing low PDGF-R $\alpha$ expression in a subset of microvessels in an uninjured myocardium (A) and strongly increased PDGF-R $\alpha$ expression in sprouting vessels in the border region of a myocardial infarct, from where vessels revascularize the infarct (B and C). C shows a detail of PDGF-R $\alpha$ expression in microvessels (arrows). (D) Upper and middle panels: immunoprecipitation and subsequent Western blotting for PDGF-R $\alpha$ (upper) and pTyr (middle) showed that PDGF-R $\alpha$ was upregulated in the ischemic myocardial regions bordering the infarct where vessels start to grow. Note also that PDGF-R $\alpha$ was activated more in the borders than the normal (nonischemic) regions and maximally after PDGF-CC treatment. Lower panel: Coomassie staining revealing comparable loading. $(\mathbf{E}$ and $\mathbf{F})$ PDGF-CC protein treatment increased $\mathrm{TM}^{+}(\mathrm{E})$ and $\mathrm{SMA}^{+}(\mathbf{F})$ vessel density in the infarcted areas in a dose-dependent manner. ${ }^{*} P<0.05$ vs. vehicle. (G-I) TM immunostaining of myocardial vessels, revealing increased vessel densities after PDGF-CC treatment. $(\mathbf{J}-\mathbf{L}) \alpha-S M A$ immunostaining of myocardial vessels, revealing increased vessel densities after PDGF-CC treatment. Scale bars: 50 $\mu \mathrm{m}$ in $\mathbf{A}, \mathbf{B}, \mathbf{G}-\mathbf{L}$ and $20 \mu \mathrm{m}$ in $\mathbf{C}$. ischemic myocardium or limb. We have therefore addressed these issues in the present study.

\section{Results}

PDGF-CC stimulates vessel growth and maturation in the ischemic heart. We used an established mouse model of coronary artery ligation to assess whether PDGF-CC stimulates revascularization of the ischemic myocardium. By immunostaining (Figure 1, A-C) as well as RNAse protection analysis and in situ hybridization (data not shown), PDGF-R $\alpha$ was detectable in a subset of myocardial vessels at baseline conditions, but its expression was significantly upregulated in the ischemic border zones surrounding the infarcts from which new vessels infiltrated the infarct. PDGF-R $\alpha$ was expressed in ECs and SMCs but likely also in additional cell types, putatively identified as myofibroblasts and inflammatory cells. Immunoprecipitation and subsequent immunoblotting of extracts from the microdissected infarct borders further confirmed that PDGF-R $\alpha$ levels were upregulated in the angiogenic regions (Figure 1D). Moreover, PDGF-R $\alpha$ was, as assessed by immunoblotting of phosphotyrosine (pTyr) residues after immunoprecipitation, highly activated in these angiogenic border zones (Figure 1D).

To examine whether PDGF-CC stimulated revascularization of the ischemic myocardium, we delivered recombinant human PDGF-CC via an osmotic minipump. PDGF-CC increased the levels of active PDGF-R $\alpha$ in the border region (Figure 1D). After 7 days, angiogenesis in the ischemic area was quantified by counting the number of EC-lined vessels after immunolabeling with anti-thrombomodulin (anti-TM) antibodies. Vessel maturation ("arteriogenesis") was evaluated by counting the arterioles immunoreactive for $\alpha$-SMA. At $1.5 \mu \mathrm{g} / \mathrm{d}$, PDGF-CC minimally affected the $\mathrm{TM}^{+}$vessel density (Figure 1, E, G, and $\mathrm{H}$ ) but increased, by 1.4 -fold, the number of $\alpha$-SMA ${ }^{+}$arterioles (Figure 1, $\mathrm{F}, \mathrm{J}$, and $\mathrm{K}$ ). When 3-fold-higher dose was used, PDGF-CC significantly stimulated angiogenesis (Figure 1, E and I) and arteriogenesis (Figure 1, F and L). No signs of hemorrhage, edema, or fibrosis were observed in PDGF-CC-treated hearts. These new vessels were functional, as perfusion of the ischemic myocardial region was significantly increased (blood flow: $1.6 \pm 0.2 \mathrm{ml} / \mathrm{min} / \mathrm{g}$ in control vs. $2.2 \pm 0.2 \mathrm{ml} / \mathrm{min} / \mathrm{g}$ after treatment with $4.5 \mu \mathrm{g} / \mathrm{d}$ PDGF-CC; $n=7-9 ; P<0.05)$. PDGF-CC stimulation of revascularization was restricted to the ischemic heart, as no differences were observed in vessel density in other organs (data not shown). The magnitude of the potential of PDGF-CC to stimulate revascularization of the ischemic myocardium parallels that of VEGF and PlGF (41). The mice tolerated the PDGF-CC treatment without signs of toxicity (weight loss, inactivity). Thus, PDGF-CC protein treatment promoted functional revascularization in cardiac ischemia via enhanced angiogenesis (more vessels) and arteriogenesis (more SMC coverage). The angio-/arteriogenic activity of PDGF-CC in cardiac ischemia is remarkable, since PDGF-AA, another PDGF-R $\alpha$ ligand, is weakly angiogenic or even suppresses angiogenesis $(24,38,42)$.

PDGF-CC stimulates vessel growth in the ischemic hind limb. We also evaluated whether PDGF-CC stimulated revascularization of 

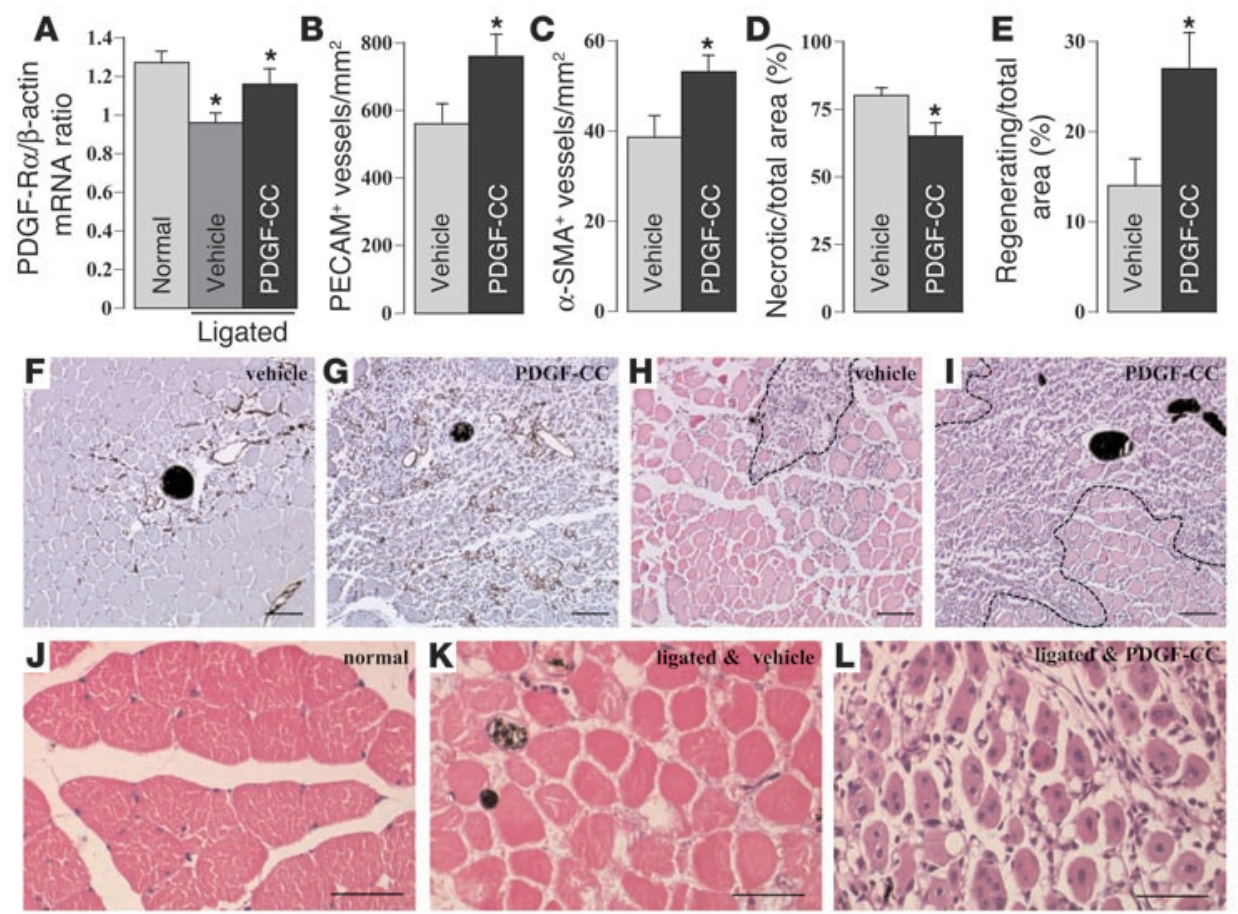

\section{Figure 2}

Therapeutic revascularization with PDGF-CC in ischemic limbs. (A) RNAse protection analysis, showing that PDGF-R $\alpha$ expression in the gastrocnemius muscle was decreased at 2 days after femoral artery ligation but restored to normal levels after PDGF-CC treatment. The ratio of the PDGF-R $\alpha$ levels (arbitrary units), normalized for $\beta$-actin levels, is shown. (B and C) PDGF-CC protein treatment increased the PECAM capillary (B) and $\alpha-S M A^{+}$arteriolar (C) density in the ischemic gastrocnemius muscle. (D and E) PDGF-CC protein treatment decreased muscle necrosis (D) and increased muscle regeneration $(E)$ in the gastrocnemius muscle at 7 days after femoral artery ligation. Areas are percentage of total muscle area. ${ }^{*} P<0.05(\mathbf{A}-\mathbf{E})$. ( $\mathbf{F}$ and $\left.\mathbf{G}\right)$ Compared with vehicle $(\mathbf{F})$, PDGF-CC protein treatment increased the density of PECAM vessels in the regenerating areas of the ischemic gastrocnemius muscle $(\mathbf{G})$. No signs of edema, hemorrhage. or fibrosis were observed. (H and $\mathbf{I})$ H\&E staining, showing larger areas of regenerating myocytes (small cells with central nuclei) after PDGF-CC treatment (I) than after treatment with vehicle $\mathbf{( H )}$. The regions containing regenerating myocytes are surrounded by a dashed black line in both panels. (J-L) Higher magnification of H\&E-stained sections of a normal gastrocnemius muscle (J); ischemic muscle, treated with vehicle, containing numerous necrotic ghost myocytes, and few blood vessels (K); ischemic muscle, treated with PDGF-CC, containing numerous regenerating myocytes with a central nucleus and numerous blood vessels (L). Values are mean \pm SEM of at least 15 mice. The lumen of the arterioles is filled with dark bismuth gelatin in F-L. Scale bars: $50 \mu \mathrm{m}$.

ischemic hind limbs, using an established model (41). We first quantified PDGF-R $\alpha$ expression in the gastrocnemius muscle, which becomes ischemic after ligation of the femoral artery. Two days after ligation, when a fraction of myocytes died due to ischemic necrosis, PDGF-R $\alpha$ transcript levels decreased to $76 \%$ of those found in normal muscles (Figure 2A). However, compared with vehicle, a daily treatment with $4.5 \mu \mathrm{g}$ PDGF-CC upregulated PDGF-R $\alpha$ expression at day 2 after ligation and almost completely restored its expression levels to values found in the unligated control muscle (Figure 2A). Revascularization of the ischemic gastrocnemius muscle after femoral artery ligation was evaluated after 1 week of continuous delivery of $4.5 \mu \mathrm{g} / \mathrm{d}$ PDGF-CC. Treatment with PDGF-CC increased both the formation and maturation (via coverage by SMCs) of new blood vessels (Figure 2, B, C, F, and G). Moreover, PDGF-CC enhanced skeletal muscle regeneration (Figure 2, $\mathrm{E}$ and $\mathrm{H}-\mathrm{L}$ ) and, as a result, also reduced the extent of ischemic muscle necrosis (Figure 2, D and $\mathrm{H}-\mathrm{L}$ ), which suggests that muscle regeneration and angiogenesis might be linked. PDGF-CC also enlarged the second-generation collateral side branches in the adductor muscle (lumen area: $680 \pm 40$ $\mu \mathrm{m}^{2}$ after saline vs. $920 \pm 100 \mu \mathrm{m}^{2}$ after PDGF-CC; $n=10 ; P=0.05$ ). No signs of hemorrhage, edema, or fibrosis were observed in the
PDGF-CC-treated limbs. Thus, PDGF-CC stimulates revascularization in mouse models of both heart and limb ischemia.

PDGF-CC mobilizes endothelial progenitors. To examine how PDGFCC stimulates vessel growth, we studied the recruitment of endothelial progenitor cells (EPCs). EPC mobilization was quantified by counting the number of cells positive for 1,1'-dioctadecyl-3,3,3',3'-tetramethylindocarbocyanine perchlorate-acetylated LDL (DiI-ac-LDL)/isolectin B4 at 3 weeks after after spleen mononuclear cells were plated. Evaluated at the time point of 3 weeks, only late-outgrowth EPCs, but not surviving sloughed-off ECs, are selectively assayed (43). In baseline conditions, PDGF-CC did not mobilize EPCs (Figure 3A), consistent with our observation that PDGF-CC only affected vessel growth in ischemic tissues. However, after femoral artery ligation, administration of $4.5 \mu \mathrm{g} / \mathrm{d}$ PDGF-CC significantly augmented EPC mobilization - the effect being maximal at 2 days (Figure 3A). We then compared the relative efficacy of the different PDGF forms side-byside by administering the same dose of PDGF-AA, -BB, or -CC $(4.5 \mu \mathrm{g} / \mathrm{d})$ to ligated mice and quantifying the number of mobilized EPCs. Both PDGF-AA and -CC mobilized EPCs at 2 days after limb ischemia, while PDGF-BB only insignificantly affected EPC 
A

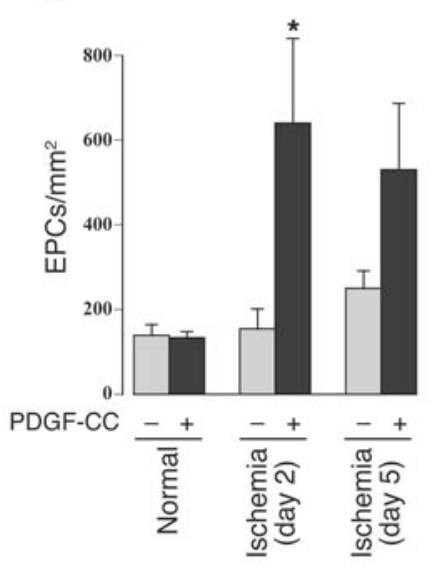

B
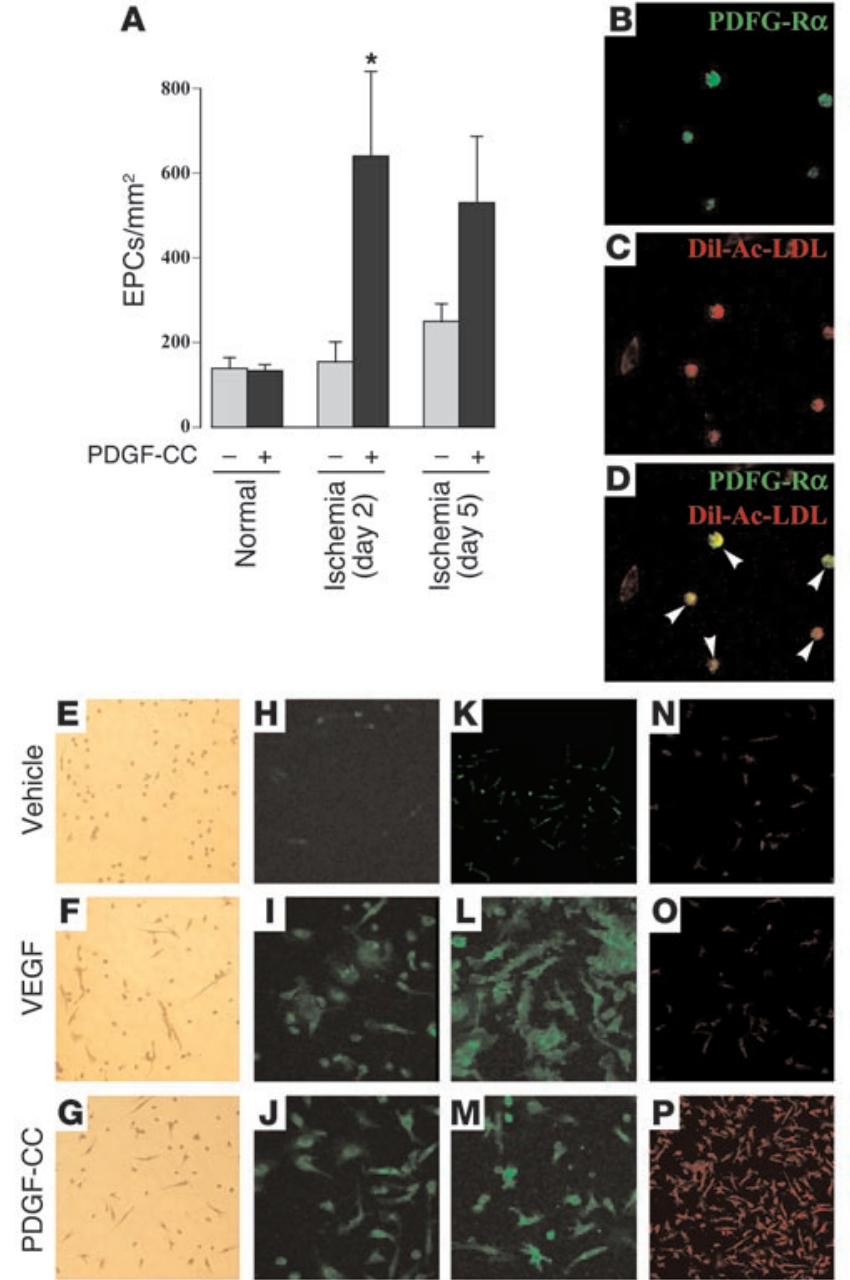

CD144

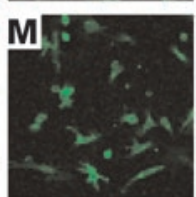

CD31
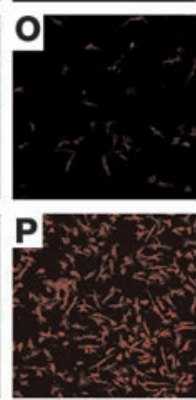

$\alpha-\mathrm{SMA}$

mobilization. The numbers of EPCs per millimeter squared were: $490 \pm 140$ after treatment with vehicle, $940 \pm 130$ after PDGF-AA $(n=12 ; P<0.05), 640 \pm 90$ after PDGF-BB $(n=12 ; P=\mathrm{NS})$, and $990 \pm 140$ after PDGF-CC $(n=12 ; P<0.05)$. In addition, late-outgrowth EPCs expressed PDGF-R $\alpha$ as revealed by real-time quantitative RT-PCR analysis ( $25 \pm 5 \mathrm{mRNA}$ copies PDGF-R $\alpha$ per 1,000 mRNA copies $\beta$-actin; $n=3$ ). Moreover, labeling of the cultured cells with the anti-PDGF-R $\alpha$ antibody revealed that the round, cobblestone-like Ac-LDL ${ }^{+}$EPCs expressed detectable levels of PDGF-R $\alpha$ (Figure 3, B-D). Thus, PDGF-CC enhanced EPC mobilization in conditions of tissue ischemia, thereby providing a source of ECs for building new blood vessels.

PDGF-CC promotes differentiation of bone marrow progenitors. To investigate the potential role of PDGF-CC in the differentiation of bone marrow progenitors into vascular cells, we cultured human bone marrow-derived $\mathrm{AC} 133^{+} \mathrm{CD} 34^{+}$cells - a population enriched for stem/progenitor cells (44) - and stimulated them with PDGF-CC, using VEGF as control (50 $\mathrm{ng} / \mathrm{ml}$ each). After 2 weeks, both PDGF-CC and VEGF enhanced the adherence of these cells - a prerequisite for anchorage-dependent cell differentiation and growth (adherent cells, \% of vehicle control: $230 \% \pm 30 \%$ for VEGF; $180 \% \pm 25 \%$ for PDGF-CC; $P<0.05$ ). However, the 2 factors markedly differed in their ability to induce the commitment of

\section{Figure 3}

Effects of PDGF-CC on endothelial progenitors. (A) PDGF-CC treatment increased EPC mobilization from day 2 to day 5 after hind limb ischemia but did not affect EPC mobilization in normal conditions. ${ }^{\star} P<0.05$. Values are mean \pm SEM of 10 mice. (B-D) Double labeling of EPCs for PDGF-R $\alpha$ (green in B) and Dil-ac-LDL (red in C), showing coexpression (yellow in D). (E-P) After 2 weeks of stimulation, both PDGF-CC and VEGF induced the expression of EC surface markers CD144 (VE-cadherin) and CD31 (PECAM), while vehicle-treated cells remained negative. Only PDGF-CC induced prominent $\alpha$-SMA expression, while cells treated with VEGF or vehicle displayed background levels of $\alpha$-SMA expression. Unstained cells in $\mathbf{E}-\mathbf{G}$ show that both VEGF and PDGF-CC promoted stem/progenitor cell adherence.

these progenitors into either ECs or SMCs. Both PDGF-CC and VEGF induced the expression of the EC surface markers vascular endothelial cadherin (VE-cadherin) (CD144; Figure 3, H-J) and CD31 (platelet endothelial cell adhesion molecule [PECAM]; Figure 3, K-M). Though these progenitors cells expressed PDGF-R $\alpha$ (RT-PCR analysis; data not shown), some of the effect of PDGF-CC to induce an EC phenotype may be attributable to the upregulation of VEGF by PDGF-CC in the progenitor cell population (ratio of VEGF to TATA box-binding protein mRNA copies: $0.43 \pm 0.09$ in control vs. $0.81 \pm 0.08$ after treatment with PDGF-CC; $n=5$; $P<0.05)$. Interestingly, only PDGF-CC additionally induced the expression of the SMC marker $\alpha$-SMA, which indicates that these cells had acquired a characteristic SMC phenotype (Figure 3, N and P). Notably, this effect of PDGF-CC was specific, as VEGF-treated cells did not become $\alpha$-SMA positive (Figure 3O). Even though SMCs release VEGF in response to PDGF-CC (see below), most of the PDGF-CC-treated cells became $\alpha$-SMA positive and lost their expression of VE-cadherin and CD31 by 4 weeks of stimulation. VEGF-treated cells, instead, were still VE-cadherin and CD31 positive but remained $\alpha$-SMA negative (data not shown). Thus, PDGF-CC initially induced bone marrow progenitor cells to differentiate into ECs - eventually, after long-term treatment, yielding cells with an SMC-like phenotype.

PDGF-CC promotes EC migration and microvessel sprouting. Expression of PDGF-R $\alpha$ on ECs has been observed in some studies but not in others $(21,22,25-27,29-34)$. In addition, little is known about the functional consequences of PDGF-R $\alpha$ signaling in these cells. We therefore analyzed the effect of PDGF-AA, -BB, and $-\mathrm{CC}$ on migration and proliferation of several EC types, using various assays. As ECs are known to express low levels of PDGF-R $\alpha$, we enriched cell lysates for PDGF-Rs by using wheat germ agglutinin-sepharose (WGA-sepharose) before immunoblotting. Both human microvascular ECs (HMVECs) and human umbilical venous ECs (HUVECs) expressed detectable levels of PDGF-R $\alpha$, which was tyrosine phosphorylated upon PDGF-CC treatment (data not shown), whereas PDGF-R $\beta$ was undetectable (Figure 4A). In a first assay, growth-arrested confluent HMVEC monolayers were wounded with a rubber policeman and then incubated for 20 hours in serum-free medium containing the indicated ligand. Under those conditions, VEGF and PDGF-CC strongly stimulated migration of HMVECs (Figure 4B). PDGF-AA had a slight effect, whereas PDGF-BB was completely ineffective (Figure 4B). The chemotactic effect of PDGF-CC was not restricted to HMVECs only, as PDGF-CC also enhanced the migration of bovine aorta ECs (BAECs; data not shown). The chemotactic effect of PDGF-CC was confirmed using HUVECs in the Boyden 
A

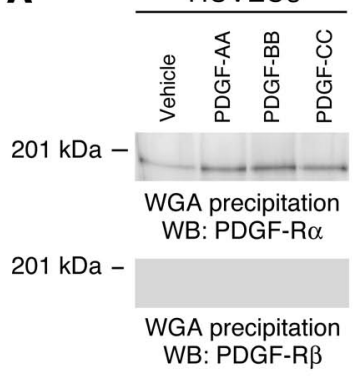

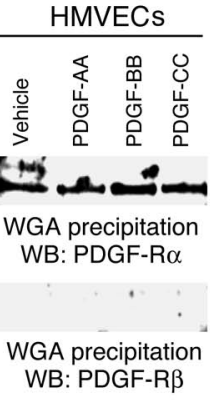

B
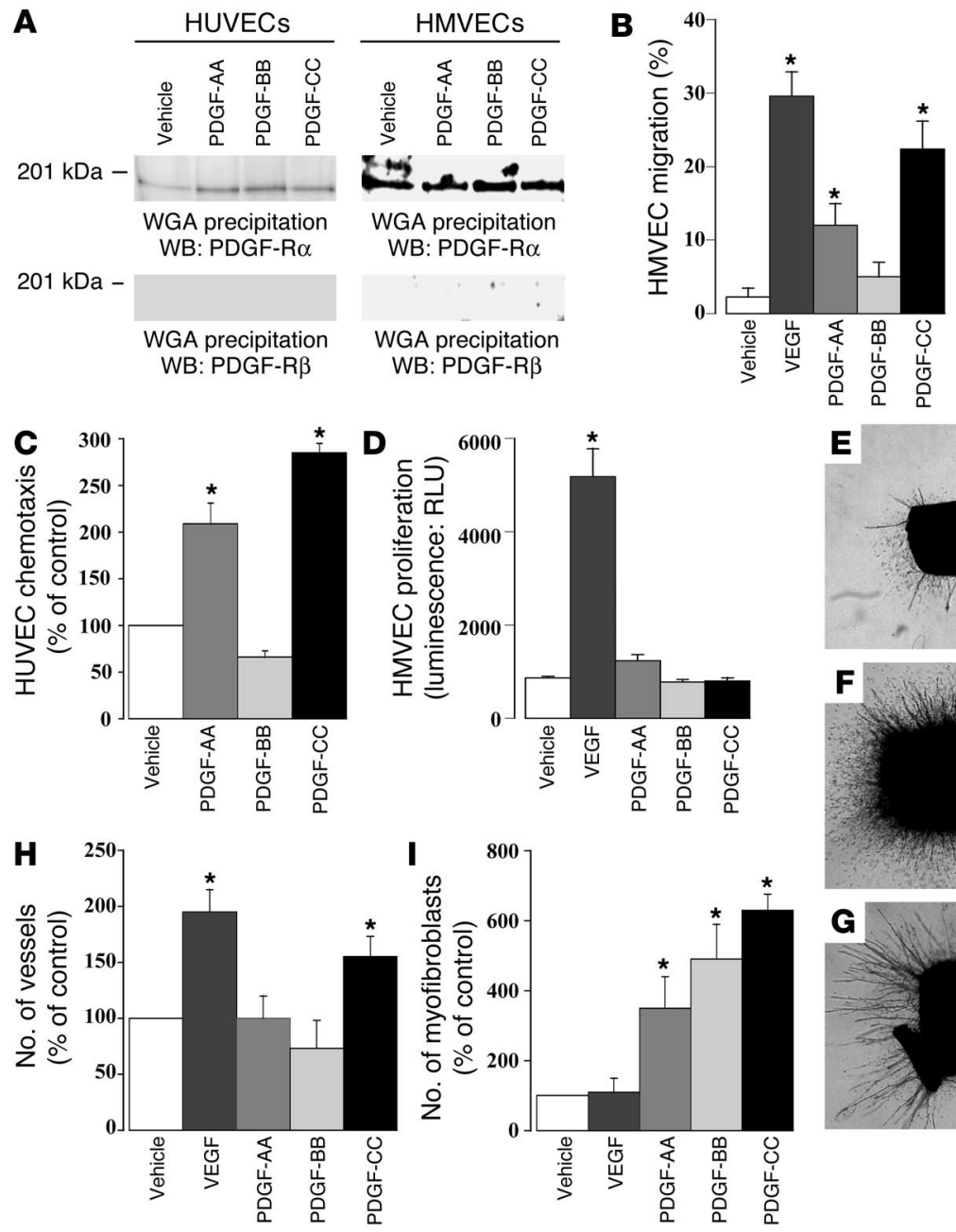
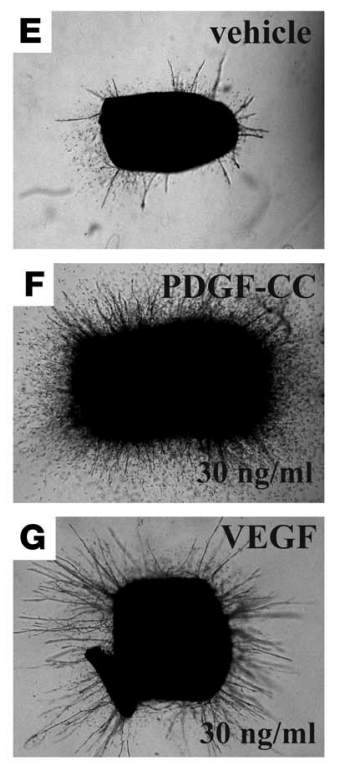

\section{Figure 4}

Effect of PDGF-CC on EC migration and outgrowth of microvessels. (A) WGA precipitation and subsequent immunoblotting of extracts of control and ligand-stimulated HUVECs and HMVECs, revealing detectable expression of PDGF-R $\alpha$ but not of PDGF-R $\beta$. By immunoblotting, PDGF-R $\alpha$ was tyrosine phosphorylated in both cell lines (data not shown). (B and C) In the scrape wound assay (B), PDGF-CC stimulated HMVEC migration with a potency similar to that of VEGF, while PDGF-AA and -BB had an intermediate or no effect, respectively. In the Boyden chamber assay (C), both PDGF-AA and -CC induced HUVEC chemotaxis, while PDGF-BB was inactive. (D) None of the PDGFs affected HMVEC proliferation, while VEGF potently promoted cell proliferation. ${ }^{*} P<0.05$. Values are mean \pm SEM. RLU, relative luminescence units. (E-G) Micrographs of aortic rings, displaying microvascular sprouts and perivascular cells. Compared to vehicle (E), PDGF-CC enhanced the outgrowth of both microvascular sprouts and fibroblast-like cells (F), while VEGF stimulated microvascular outgrowth (G). ( $H$ and $\mathbf{I}$ In the aortic ring assay, PDGF-CC and VEGF induced microvessel outgrowth, while PDGF-AA and -BB were inactive $(\mathbf{H})$. Each PDGF form stimulated myofibroblast outgrowth from the aortic ring, but PDGF$\mathrm{CC}$ was more potent than PDGF-BB and PDGF-AA, whereas VEGF was inactive (I). $P<0.05$ ( $\mathbf{H}$ and $\mathbf{I}$ ). WB, Western blot. chamber assay (Figure 4C). PDGF-AA also stimulated endothelial migration, while PDGF-BB was again ineffective (Figure 4C).

These findings were further extended by analyzing in the Boyden chamber chemotaxis of porcine aortic ECs (PAECs) stably overexpressing PDGF-R $\alpha$ (PAEC/R $\alpha$ ) or PDGF-R $\beta$ (PAEC/R $\beta)$ (45). At $125 \mathrm{ng} / \mathrm{ml}$, PDGF-CC was more potent than PDGF-AA in stimulating chemotaxis of PAEC/R $\alpha$, whereas PDGF-BB had no effect (chemotactic response, $\%$ of vehicle: $260 \% \pm 29 \%$ for PDGF-AA; $92 \% \pm 11 \%$ for PDGF-BB, and $610 \% \pm 30 \%$ for PDGF-CC; $P<0.05$, PDGF-AA vs. PDGF-CC). As expected, PDGF-AA and -CC failed to affect PAEC/R $\beta$ (chemotactic response, $\%$ of vehicle: $111 \% \pm 5 \%$ for PDGF-AA; $101 \% \pm 30 \%$ for PDGF-CC), while PDGF-BB induced a strong chemotactic response (\% of vehicle: $422 \% \pm 22 \%$ for PDGF-BB; $P<0.05$ vs. control). However, none of the PDGFs (type AA, BB, or CC) affected proliferation of HMVECs (Figure 4D), BAECs, or HUVECs (data not shown), in agreement with the previous observation that PDGF-R $\alpha$ does not transmit mitogenic signals in response to PDGF-AA in ECs (25). VEGF, instead, highly stimulated EC proliferation (Figure 4D).

We also tested the effect of PDGF-CC on cultured aortic rings, as this assay allows assessing the outgrowth of microvessels from an intact vessel in vitro (46). After 9 days of culturing the rings with or without $30 \mathrm{ng} / \mathrm{ml}$ ligand, the microvessels and the perivascular myofibroblasts, growing out of the aorta, were counted. Only PDGF-CC, but not PDGF-AA or -BB, stimulated microvessel outgrowth (Figure 4, E, F, and H). Each PDGF form did, however, increase myofibroblast outgrowth, but PDGF-CC was the most potent (Figure 4I). VEGF only stimulated microvessel growth, without affecting myofibroblast emigration (Figure $4, \mathrm{G}-\mathrm{I})$. Vessel outgrowth in response to PDGF-CC was largely blocked (i.e., by $80 \%$ ) by supplementation of a neutralizing anti-VEGF antibody, which indicates that at least some of the angiogenic effect of PDGF-CC in this assay was mediated via VEGF release. Indeed, following stimulation with $50 \mathrm{ng} / \mathrm{ml}$ PDGF-CC, cultured SMCs released increased amounts of VEGF in the conditioned medium $\left(24 \pm 1 \mathrm{pg} / 10^{5}\right.$ cells $/ 24 \mathrm{~h}$, after treatment with control vs. $35 \pm 1 \mathrm{pg} / 10^{5}$ cells $/ 24 \mathrm{~h}$, after treatment with $50 \mathrm{ng} / \mathrm{ml}$ PDGF-CC; $n=4 ; P<0.05)$. This effect was specific, as $50 \mathrm{ng} / \mathrm{ml}$ PDGF-AA failed to stimulate VEGF expression $\left(22 \pm 1 \mathrm{pg} / 10^{5}\right.$ cells $/ 24 \mathrm{~h} ; n=4 ; P=\mathrm{NS}$ vs. control). Thus, PDGF-CC is capable of stimulating angiogenesis via direct and indirect effects on ECs.

Tyrosine phosphorylation of PDGF-R $\alpha$ in response to PDGF-CC. Although PDGF-AA, -BB, and -CC bind PDGF-R $\alpha$, these ligands did not always induce an identical response, for example, in EPC recruitment, EC migration, microvessel and myofibroblast outgrowth from the aortic ring, or induction of VEGF release. We therefore studied whether activation of PDGF-R $\alpha$ and tyrosine 


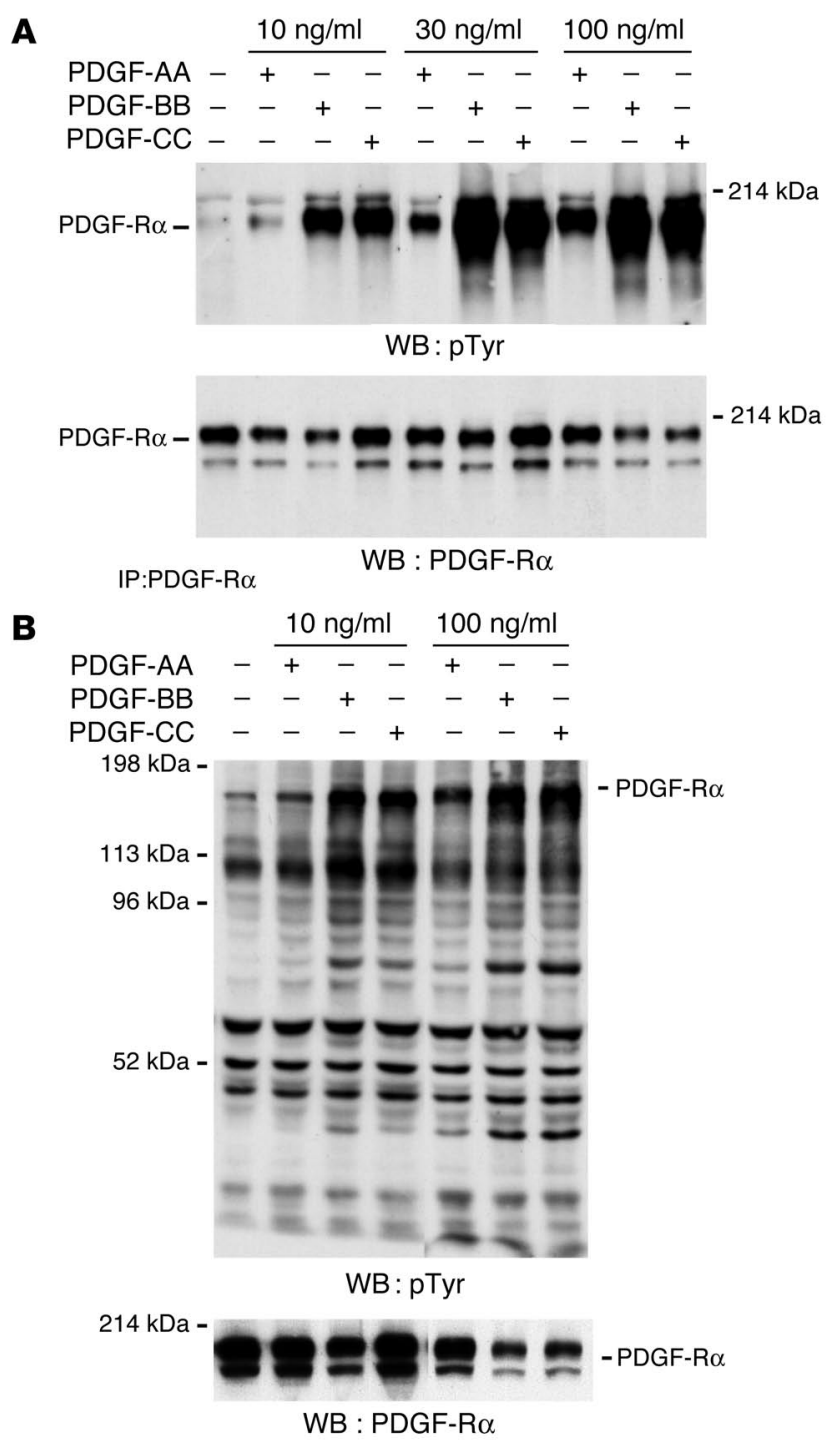

phosphorylation of downstream proteins by PDGF-AA, -BB, and -CC in ECs was different. Since PDGF-R $\alpha$ levels in primary ECs are insufficient to permit reliable detection of PDGF-R $\alpha$ tyrosine phosphorylation, we used PAEC/R $\alpha$ (45). After stimulation of these cells with each ligand at 10,30 , and $100 \mathrm{ng} / \mathrm{ml}$ for 10 minutes, equal amounts of cell extracts were immunoprecipitated with anti-PDGF-R $\alpha$ antibodies and subsequently immunoblotted with pTyr-specific and PDGF-R $\alpha$-specific antibodies. Specific bands were then densitometrically quantified, and the PDGF-R $\alpha-$ pTyr/PDGF-R $\alpha$ ratio was calculated. At all concentrations tested, PDGF-AA stimulated tyrosine phosphorylation of PDGF-R $\alpha$ less than PDGF-BB and -CC (Figure 5A). After quantification, the PDGF-R $\alpha-$ pTyr/PDGF-R $\alpha$ ratio increased $3.3 \pm 0.5-, 4.6 \pm 0.2$-, and $5.6 \pm 1.0$-fold when cells were treated with 10,30 , and 100 ng/ml PDGF-AA, respectively $(n=3-7 ; P<0.05)$, while this ratio increased $9 \pm 1$-, $13 \pm 3$-, and $23 \pm 5$-fold after stimulation with 10 , 30 , and $100 \mathrm{ng} / \mathrm{ml}$ PDGF-CC, respectively $(n=3-7 ; P<0.05$ vs. PDGF-AA by ANOVA with Bonferroni correction). Corresponding values for PDGF-BB were $18 \pm 2$-, $20 \pm 2$-, and $44 \pm 8$-fold, respectively ( $n=3 ; P<0.05$ vs. PDGF-AA by ANOVA). Thus, at least in PDGF-R $\alpha$-expressing PAECs, PDGF-BB and -CC induced

\section{Figure 5}

Tyrosine phosphorylation of PDGF-R $\alpha$ and downstream proteins in response to PDGF-CC. (A) Immunoprecipitation of equal amounts of $\mathrm{PAEC} / \mathrm{R} \alpha$ extracts for PDGF-R $\alpha$ and subsequent immunoblotting for pTyr, revealing a higher degree of PDGF-R $\alpha$ tyrosine phosphorylation after 10-minute stimulation with PDGF-BB or -CC than with PDGF-AA. The lower panel shows an immunoblot of PDGF-R $\alpha$, displaying the amount of receptor present in each sample. (B) Immunoblotting of equal amounts of PAEC/R $\alpha$ extracts for pTyr after stimulation with PDGF-AA, -BB, or -CC for 10 minutes, revealing that a similar set of proteins (judged on the basis of their molecular weight) was phosphorylated but that, in general, the phosphorylation signals were stronger in response to PDGF-BB and -CC than PDGF-AA. The lower panel shows an immunoblot of PDGF-R $\alpha$, displaying the amount of receptor present in each sample. For both $\mathbf{A}$ and $\mathbf{B}$, though equal amounts of cell lysate were immunoprecipitated (A) and/or loaded (A and B), PDGF-R $\alpha$ levels differed among the various lanes, presumably reflecting effects on receptor internalization and degradation. PDGF-R $\alpha$ is present as a doublet due to differences in glycosylation.

a higher degree of PDGF-R $\alpha$ activation than PDGF-AA. We also analyzed whether PDGF-AA, -BB, and -CC affected downstream signaling differently. Therefore, equal amounts of total cell lysates from PAEC/R $\alpha$, stimulated with 10 or $100 \mathrm{ng} / \mathrm{ml}$ PDGF-AA, -BB, or -CC for 10 minutes, were immunoblotted using the PY99 pTyr antibody. At least when identifying the phosphorylated proteins on the basis of their molecular weight, PDGF-AA, -BB, and -CC seemed to stimulate tyrosine phosphorylation of a similar panel of downstream targets (Figure $5 \mathrm{~B}$ ). However, at both concentrations, PDGF-BB and -CC generally induced stronger phosphorylation signals than did PDGF-AA.

\section{Discussion}

Study of the role of PDGF-CC in angiogenesis in vivo has been limited. One study reported that PDGF-CC has no effects on EC outgrowth from the aorta (5), while another documented effects of PDGF-CC on microvessel outgrowth and branching in the mouse cornea and chicken embryo (35). From these studies, PDGF-CC seemed to affect ECs indirectly via effects on SMCs $(1,5,39)$, in part by inducing the release of VEGF (40). Direct effects of PDGF-CC on ECs or their progenitors have not been reported thus far. Here, we show that PDGF-CC stimulates the recruitment of endothelial progenitors from the bone marrow. Numerous studies have documented that adult bone marrowderived progenitor cells can contribute to the revascularization of and, thereby, facilitate the regeneration and functional recovery of the ischemic limb and heart. However, the signals that trigger their mobilization and induce their differentiation into more specialized vascular cells remain more enigmatic. In the present study, we found that PDGF-CC mobilizes EPCs within the first 2 days and up to 5 days after tissue ischemia. This is precisely the time window within which new blood vessels start to grow in these ischemic tissues.

EPCs were found to express PDGF-R $\alpha$ mRNA transcript and protein, which indicates that they respond to PDGF-CC. Our findings obviously do not exclude the possibility that PDGF-CC may also affect EPC mobilization indirectly via upregulation of additional mobilization signals, such as VEGF. In fact, many cell types, including bone marrow-derived EPCs and SMCs (the present study), fibroblasts (data not shown and ref. 47), and tumor cells (40) release VEGF in response to PDGF-CC, which suggests 
that direct and indirect effects might be involved. As PDGF-CC only stimulated the mobilization of EPCs after induction of limb ischemia, additional recruitment signals, induced by ischemia, are likely involved.

PDGF-BB stimulates vascular progenitors to acquire an SMC phenotype in the embryo and adult, but the effect of PDGF-CC had never been studied. Here, we show that PDGF-CC promoted $\mathrm{AC} 133^{+} \mathrm{CD} 34^{+}$progenitors to differentiate into cell types with EC or SMC markers. VEGF, instead, only promoted these progenitors to differentiate into ECs. PDGF-CC initially promoted the differentiation of both lineages but subsequently, after prolonged treatment, favored the differentiation of SMCs. The activity of PDGF-CC to induce an endothelial phenotype may be due to direct effects on these progenitors (as they express PDGF-R $\alpha$ ) but also to indirect effects via release of VEGF by the progenitors themselves. Our data do not allow us to distinguish between the possibilities that PDGF-CC stimulated the transdifferentiation of ECs to SMCs or that it enhanced the selection of SMCs starting from a single population of a common vascular progenitor or from a mixed population of endothelial and SMC progenitors. A possible implication of these findings is that when only VEGF is present, bone marrow-derived progenitors will preferentially contribute to the formation of endothelial capillaries. Instead, when PDGF-CC is present, it might favor their differentiation into both ECs and SMCs and, thereby, promote not only the formation but also the stabilization of nascent vessels.

The activity of PDGF-CC is, however, not restricted to vascular progenitors only. Indeed, this growth factor also stimulated the migration of differentiated ECs - both when studied as isolated cultured cells (by scrape wounding and Boyden chamber assays) and in the aortic ring assay. Such an effect on ECs has not been reported previously. There may be several explanations for this. For instance, the fact that several studies did not detect PDGF-R $\alpha$ or $-\mathrm{R} \beta$ on cultured ECs may relate to the finding that PDGF-R expression is downregulated in culture and that PDGF-Rs are expressed only by some but not all EC types (22, 25, 26, 30, 32, 33). Moreover, detection of the low PDGF-R levels in cultured ECs requires sensitive precipitation and immunoblotting techniques. Using WGA for precipitation permitted us to detect the low levels of PDGF-R $\alpha$ on HMVECs and HUVECs. Importantly, however, PDGF-R $\alpha$ expression in cultured ECs is not an in vitro artifact, as PDGF-R $\alpha$ is present on ECs in the heart and, in particular, upregulated in inflamed and ischemic areas of new vessel growth. These findings are consistent with previous reports that PDGF-R $\alpha$ is expressed in tumor areas with active vessel growth but not in quiescent endothelium $(22,30,48)$. It thus seems that PDGF-R $\alpha$ expression is highly regulated in vivo and that such complex regulation might be preserved or lost in vitro, perhaps depending on experimental conditions such as the serum, the passage number and type of ECs, etc. Overall, the effects of PDGF-CC on ECs observed in this study suggest that PDGF-CC stimulates angiogenesis in part by affecting ECs directly.

PDGF-AA, -BB and-CC all bind PDGF-R $\alpha$, yet they did not induce identical biological responses. For instance, PDGF-AA and -CC mobilized EPCs more efficiently than PDGF-BB, while PDGF-AA and $-\mathrm{BB}$ were less potent than PDGF-CC in stimulating migration of ECs in wounded monolayers. Furthermore, PDGF-CC was more effective than PDGF-AA and -BB in stimulating microvessel outgrowth and myofibroblast emigration from the aorta. How can these differences be explained? One possible explanation is that
PDGF-BB and -CC, but not PDGF-AA, are capable of transmitting signals via PDGF-R $\beta$, when PDGF-R $\beta$ is coexpressed with PDGF-R $\alpha$ in ECs. PDGF-R $\beta$ was, however, undetectable in our ECs, and thus the effect of PDGF-BB or -CC would not be expected to be mediated via PDGF-R $\alpha \beta$ or $-\mathrm{R} \beta \beta$. These findings therefore raise the question of whether PDGF-AA, -BB, and -CC differ in their ability to stimulate, quantitatively or qualitatively, PDGF-R $\alpha-\mathrm{a}$ provocative question that cannot be conclusively addressed by our present findings. When PAEC/R $\alpha$ was used, PDGF-AA stimulated tyrosine phosphorylation of PDGF-R $\alpha$ and downstream proteins less than PDGF-BB or -CC, but it remains to be established whether this is also true for nontransfected ECs expressing physiological levels of PDGF receptors. Alternatively, we also cannot exclude the possibility that yet another coreceptor is involved in the response to some of the PDGFs. Whatever the mechanism, the distinct complementary biological activities of PDGF-AA, $-\mathrm{BB}$, and -CC may further help to explain why loss of $p d g f-r \alpha$ causes a more severe phenotype than that caused by elimination of the pdgf-a gene alone $(1,49)$ and why only the combined loss of the $p d g f-a$ and $-c$ genes phenocopied the loss of $p d g f-r \alpha$ (50).

PDGF-CC treatment mobilized endothelial progenitors and increased the vessel density and blood perfusion in the ischemic heart and limb but did not affect quiescent vessels in other organs. Although PDGF-CC enlarged the second-generation side branches of the collateral vessels in the adductor muscle, this growth factor has, overall, a less dramatic effect on the remodeling of the preexisting collaterals in the upper limb region after femoral artery ligation than, for instance, bFGF, PlGF, or GM-CSF $(41,51)$. However, the molecular and cellular mechanisms of the growth of collateral vessels are quite distinct from those determining the formation of new capillaries and their maturation by coverage with SMCs. In particular, not ischemia but shear stress-induced recruitment of monocytes/macrophages is well known to play a critical role in initiating collateral growth in the upper hind limb (51), and PDGF-CC does not affect their recruitment (data not shown). Since only the lower, but not the upper, limb is ischemic after femoral artery ligation, PDGF-CC seems to be more involved in ischemia-dependent angiogenesis than in the shear stress-induced collateral vessel remodeling. Our findings that PDGF-CC is more effective in ischemic than nonischemic tissues do not exclude the possibility that PDGF-CC might affect vessel growth in nonischemic tissues. PDGF-CC did, for instance, stimulate angiogenesis in the chick embryo (35). But in the adult, PDGF-CC has thus far only been documented to enhance angiogenesis in the avascular - and therefore ischemic - cornea (35), which supports our present findings.

Despite the initial lack of success in clinical trials, therapeutic revascularization of ischemic tissues is still an outstanding and medically important goal. Forming new blood vessels via administration of a single angiogenic factor has, apparently, been more challenging than initially considered. Of the members of the PDGF family, only PDGF-BB has been evaluated thus far. When administered alone, PDGF-BB may cause vessel regression due to endothelial apoptosis as a result of impaired mural cell recruitment $(15,16,18)$. When coadministered with FGF-2 or VEGF, PDGF-BB promotes the formation of more numerous and stable vessels (16-18). In comparison, administration of PDGF-CC alone sufficed to stimulate new vessel growth and maturation, but it remains to be evaluated whether coadministration with other angiogenic factors will not induce a greater response. As exces- 
sive and protracted overexpression of PDGF-CC to levels many fold higher than used in the present study may be implicated in organ fibrosis (e.g., heart, liver) via uncontrolled stimulation of fibroblasts $(47,52)$, caution is warranted to carefully optimize the dose and duration of the administration of this growth factor in order to selectively induce vessel growth and maturation without causing profibrotic effects.

In conclusion, PDGF-CC may stimulate vessel growth in various ways: apart from inducing the release of VEGF, it also mobilizes vascular progenitors and promotes their differentiation into ECs and SMCs, stimulates EC migration, and affects SMCs. These effects of PDGF-CC on vascular and muscle regeneration, together with its safety profile and activity in ischemic conditions, may have implications for developing novel strategies for the treatment of heart and limb ischemia.

\section{Methods}

Mouse ischemia models. Myocardial and hind limb ischemia mouse models, protein delivery, blood flow measurements, histology, and morphometric quantification of vessel densities were all performed as described (41). PDGF-CC was produced in-house as described (1). Gastrocnemius muscles were harvested 7 days after femoral artery ligation, and sections were analyzed after H\&E staining or immunostaining for the EC marker CD31 (rat anti-CD31; BD Biosciences - Pharmingen). Vessel densities and tissue necrosis/regeneration were morphometrically analyzed using KS300 image analysis software (Carl Zeiss Inc.). PDGF-R $\alpha$ transcript levels were detected by in situ hybridization, as described (53). PDGF-R $\alpha$ protein was visualized on paraformaldehyde-fixed cryosections of the heart using a PDGF-R $\alpha$-specific antibody (rabbit anti-human PDGF-R $\alpha$, called R7, raised against the C-terminal amino acid residues 1066-1084 of PDGF-R $\alpha$; ref. 54). Procedures involving experimental animals were approved by the Institutional Animal Care and Research Advisory Committee of the Univeristy of Leuven, Leuven, Belgium (P02047 and P02046).

EPC mobilization assay. Mice were treated with vehicle (PBS), PDGF-AA (R\&D Systems), PDGF-BB (54), or PDGF-CC (1) (4.5 $\mu \mathrm{g} / \mathrm{d}$ ) immediately after femoral artery ligation using osmotic minipumps and, after 2 or 5 days, spleen mononuclear cells were plated out for EPC analysis as described (55). Late-outgrowth EPCs (after 3 weeks of culture) were identified by metabolic uptake of DiI-ac-LDL (Molecular Probes) and positive staining of Alexa 488labeled isolectin B4 (Molecular Probes Inc.). EPC densities of 5 microscopic fields at $\times 200$ magnification (using a confocal microscope) were determined and averaged by an investigator blinded to the treatment.

Human bone marrow-derived $\mathrm{AC} 133^{+} \mathrm{CD} 34^{+}$cells. Enriched bone marrow-derived $\mathrm{AC} 133^{+} \mathrm{CD} 34^{+}$cells (BioWhittaker) were seeded in a 6-well collagen-coated plate (BD) and cultured for 3 days in hematopoietic growth medium (BioWhittaker) supplemented with 4\% FCS (Invitrogen Corp.), thrombopoietin (50 ng/ml), SCF (25 ng/ml), and Flt-3 ligand $(50 \mathrm{ng} / \mathrm{ml} ; \mathrm{R} \& \mathrm{D}$ Systems). The cultures were depleted of any contaminating stromal fibroblasts by subculturing, after 3 days, the nonadherent fraction of $\mathrm{AC}^{2} 33^{+} \mathrm{CD} 34^{+}$cells at $10^{5}$ cells $/ \mathrm{ml}$ in collagen-coated 12 -well plates in endothelial basal medium (BioWhittaker) containing 4\% FCS and $50 \mathrm{ng} / \mathrm{ml} \mathrm{VEGF}_{165}$ (R\&D Systems) or PDGF-CC. Growth factors were added every 2 days, and media were refreshed for $75 \%$ every 4 days. For the adherence assay, $2.5 \times 10^{4}$ nonadherent cells $/ \mathrm{ml}$ were cultured in the same condition on collagen-coated chamber slides or in 96-well plates coated with $0.3 \%$ gelatin in PBS. After 2 weeks of culture, cells were stained with Giemsa May-Grünwald reagent. The number of viable cells was estimated by ATP quantification using the CellTiter-Glo Luminescent Cell Viability Assay (Promega Corp.). For cell surface stainings, cells $\left(2 \times 10^{4} /\right.$ well $)$ cultured on collagen-coated culture slides, were fixed, permeabilized using the IntraStain Kit (DakoCytomation), and labeled with CD31-FITC (BD), CD144-FITC (BD Biosciences - Pharmingen), CD34-FITC (BD), or $\alpha$-SMA-Cy3 (Sigma-Aldrich). Labeled cells were analyzed using laser confocal immunofluorescence microscopy.

Cell migration, proliferation and aortic ring assay. To study EC migration, 2 assays were used. In the scrape wounding assay, growth-arrested confluent HMVEC or BAEC monolayers were wounded with a rubber policeman and washed with serum-free medium. Dishes were incubated for 20 hours in serum-free medium containing $50 \mathrm{ng} / \mathrm{ml} \mathrm{VEGF}_{165}$ or PDGF-AA, -BB, or -CC. The area of migrating cells was determined as percentage of the total wound area. The modified Boyden chamber assay was performed using a 48-multiwell microchemotaxis chamber (AP48; Neuro Probe Inc.). Polycarbonate filters (pore size $8 \mu \mathrm{m}$; Neuro Probe Inc.) were coated with $1 \%$ gelatin for 2 hours at $37^{\circ} \mathrm{C}$. Subconfluent cells were prepared in serum-free medium at $3 \times 10^{5}$ cells $/ 50 \mu \mathrm{l}$. The bottom wells were filled with $28 \mu \mathrm{l} /$ well of chemoattractant diluted in serum-free medium, and $50 \mu \mathrm{l}$ cell suspension/well was added to the upper chamber. After incubation at $37^{\circ} \mathrm{C}$ for 10 hours, the filters were fixed and, after Giemsa staining, the cells were counted in 10 fields per well at $\times 400$ magnification. All the experiments were carried out at least 3 times in duplicate.

To determine cell proliferation, 2 assays were used. In the first assay, cells were seeded in 96-well plates ( 5 wells per condition) and incubated with VEGF or PDGF-AA, -BB, or -CC $(50 \mathrm{ng} / \mathrm{ml})$ after serum starvation. After 7 days, viable cells were counted using the CellTiter-Glo Luminescent Cell Viability Assay. In the second assay, subconfluent HMVECs and HUVECs were trypsinized and seeded at 4,000 cells/well in $1 \%$ gelatin-coated 96-well microplates and incubated for 4 hours. After the cells had attached, the medium was replaced with serum-free medium containing different amounts of PDGF-AA, -BB, or -CC (0, 1, 5, 25, 50, 100, $200 \mathrm{ng} / \mathrm{ml})$. After incubation for 24, 48, and 72 hours, $10 \mu \mathrm{l}$ of Cell Proliferation Reagent WST-1 (Roche Diagnostics GmbH) was added to each well and incubated for 1 hour, after which the absorbance was measured at $450 \mathrm{~nm}$.

The aortic ring assay was performed as described (46). Briefly, 1-mmlong aortic rings were embedded in gels of rat tail interstitial collagen, cultured at $37^{\circ} \mathrm{C}$, supplemented with different growth factors $(50 \mathrm{ng} / \mathrm{ml})$ or anti-VEGF antibody (AF-493-NA; R\&D Systems), and analyzed after 9 days. Experiments included 3 explants per condition and were repeated at least twice. Aortic rings were photographed at $\times 25$ magnification, and the number of microvessels or myofibroblasts, emigrating from each aortic ring, was counted (46).

RNA and protein analysis. RNAse protection analysis was performed according to the manufacturer's protocol (Ambion Inc.), and mouse $\beta$-actin cDNA was used as internal control. Quantitative real-time PCR of VEGF and TATA box-binding protein was performed as described (41). Secreted VEGF protein was quantified using the Quantikine immunoassay kit (R\&D Systems) according to the manufacturer's protocol.

$P D G F$ receptor analysis. PAEC/R $\alpha$ (45) were starved overnight in serumfree medium and then stimulated with PDGF-AA, -BB, or -CC at the indicated concentration for 10 or 20 minutes. Thereafter, cells were lysed, and equal amounts of total cell lysates were immunoprecipitated using anti-PDGF-R $\alpha$ antibodies (sc431; Santa Cruz Biotechnology Inc.). Half of the immunoprecipitate was analyzed by Western blotting using antipTyr mAb (PY99; Santa Cruz Biotechnology Inc.), while the other half was used to determine the amount of immunoprecipitated receptor, using the anti-PDGF-R $\alpha$ antibody. Either HRP-conjugated goat anti-mouse immunoglobulins or HRP-conjugated goat anti-rabbit immunoglobulins (DakoCytomation) were used as secondary antibodies. Similar methods were used for analysis of PDGF-R $\alpha$ in tissues.

After overnight starvation in 0.1\% FCS, HMVECs and HUVECs (expressing lower PDGF-R levels) were treated with $50 \mathrm{ng} / \mathrm{ml}$ PDGF-AA, -BB, 
or -CC for 10 minutes, washed, and lysed. The lysates were cleared by centrifugation, and, in order to enrich for PDGF-Rs, WGA-sepharose (Amersham Biosciences) was added and incubated under rotation at $4{ }^{\circ} \mathrm{C}$ for 2 hours. After washing, the proteins were eluted by incubation with SDS sample buffer for 5 minutes at $95^{\circ} \mathrm{C}$, resolved with SDS-PAGE, and immunoblotted using the R7/TIE PDGF-R $\alpha$ antiserum (1:500 dilution) (54). Antibodies recognizing pTyr (sc-7020) and PDGF-R $\beta$ (sc-339) were from Santa Cruz Biotechnology Inc.

Statistics. Two-tailed Student's $t$ test was used for data analysis, with $P<0.05$ considered statistically significant. For cell migration assay, ANOVA/Dunnett test was used for data analysis, with $P<0.05$ considered statistically significant.

\section{Acknowledgments}

The authors thank S. Jansen, K. Maris, W.Y. Man, L. Notebaert, B. Vanwetswinkel, S. Terclavers, and S. Wyns (CTG-VIB, Leuven) and B. Åkerblom (Ludwig Institute of Cancer Research, Stockholm) for assistance. This work was supported by the European Union
(Biomed BMH4-CT98-3380); Actie Levenslijn (\#7.0019.98); Fonds voor Wetenschappelijk Onderzoek-Vlaanderen (\#G012500); the Belgian Science Policy (project no. IAP-P5/02); an unrestricted Bristol-Meyers Squibb grant; the Swedish Research Council; the Novo Nordisk Foundation; and the Karolinska Institute. M. Tjwa is an Instituut voor de aanmoediging van Innovatie door Wetenschap en Technologie in Vlaanderen research fellow.

Received for publication June 12, 2003, and accepted in revised form November 9, 2004.

Address correspondence to: Peter Carmeliet, Center for Transgene Technology and Gene Therapy, Flanders Interuniversitary Institute for Biotechnology, KULeuven, Campus Gasthuisberg, Herestraat 49, B-3000, Leuven, Belgium. Phone: 32-16-34-57-72; Fax: 32-16-34-59-90; E-mail: peter.carmeliet@med.kuleuven.ac.be.

\section{Xuri Li and Marc Tjwa contributed equally to this work.}

1. Li, X., et al. 2000. PDGF-C is a new protease-activated ligand for the PDGF alpha-receptor. Nat. Cell Biol. 2:302-309.

2. Bergsten, E., et al. 2001. PDGF-D is a specific, protease-activated ligand for the PDGF beta- receptor. Nat. Cell Biol. 3:512-516.

3. LaRochelle, W.J., et al. 2001. PDGF-D, a new protease-activated growth factor. Nat. Cell Biol. 3:517-521.

4. Heldin, C.H., and Westermark, B. 1999. Mechanism of action and in vivo role of platelet-derived growth factor. Physiol. Rev. 79:1283-1316.

5. Gilbertson, D.G., et al. 2001. Platelet-derived growth factor C (PDGF-C) a novel growth factor that binds to PDGF alpha and beta receptor. J. Biol. Chem. 276:27406-27414.

6. Resink, T.J., et al. 1990. Specific growth stimulation of cultured smooth muscle cells from spontaneously hypertensive rats by platelet-derived growth factor A-chain homodimer. Cell Regul. 1:821-831.

7. Hellstrom, M., Kalen, M., Lindahl, P., Abramsson, A., and Betsholtz, C. 1999. Role of PDGF-B and PDGFR-beta in recruitment of vascular smooth muscle cells and pericytes during embryonic blood vessel formation in the mouse. Development. 126:3047-3055.

8. Hellstrom, M., et al. 2001. Lack of pericytes leads to endothelial hyperplasia and abnormal vascular morphogenesis. J. Cell Biol. 153:543-553.

9. Hirschi, K.K., Rohovsky, S.A., Beck, L.H., Smith, S.R., and D'Amore, P.A. 1999. Endothelial cells modulate the proliferation of mural cell precursors via platelet-derived growth factor-BB and heterotypic cell contact. Circ. Res. 84:298-305.

10. Lindahl, P., and Betsholtz, C. 1998. Not all myofibroblasts are alike: revisiting the role of PDGF-A and PDGF-B using PDGF-targeted mice. Curr. Opin. Nephrol. Hypertens. 7:21-26.

11. Hoch, R.V., and Soriano, P. 2003. Roles of PDGF in animal development. Development. 130:4769-4784.

12. Guo, P., et al. 2003. Platelet-derived growth factor-B enhances glioma angiogenesis by stimulating vascular endothelial growth factor expression in tumor endothelia and by promoting pericyte recruitment. Am. J. Pathol. 162:1083-1093.

13. Furuhashi, M., et al. 2004. Platelet-derived growth factor production by B16 melanoma cells leads to increased pericyte abundance in tumors and an associated increase in tumor growth rate. Cancer Res. 64:2725-2733.

14. Sato, N., et al. 1993. Platelet-derived growth factor indirectly stimulates angiogenesis in vitro. Am. J. Pathol. 142:1119-1130.

15. Benjamin, L.E., Hemo, I., and Keshet, E. 1998. A plasticity window for blood vessel remodelling is defined by pericyte coverage of the preformed endothelial network and is regulated by PDGF- B and VEGF. Development. 125:1591-1598.

16. Richardson, T.P., Peters, M.C., Ennett, A.B., and Mooney, D.J. 2001. Polymeric system for dual growth factor delivery. Nat. Biotechnol. 19:1029-1034.

17. Hao, X., et al. 2004. Angiogenic effects of dual gene transfer of bFGF and PDGF-BB after myocardial infarction. Biochem. Biophys. Res. Commun. 315:1058-1063.

18. Cao, R., et al. 2003. Angiogenic synergism, vascular stability and improvement of hind-limb ischemia by a combination of PDGF-BB and FGF-2. Nat. Med. 9:604-613.

19. Pietras, K., et al. 2002. Inhibition of PDGF receptor signaling in tumor stroma enhances antitumor effect of chemotherapy. Cancer Res. 62:5476-5484.

20. Erber, R., et al. 2004. Combined inhibition of VEGF and PDGF signaling enforces tumor vessel regression by interfering with pericyte-mediated endothelial cell survival mechanisms. FASEB J. 18:338-340.

21. Uehara, H., et al. 2003. Effects of blocking platelet-derived growth factor-receptor signaling in a mouse model of experimental prostate cancer bone metastases. J. Natl. Cancer Inst. 95:458-470.

22. Kim, S.J., et al. 2004. Simultaneous blockade of platelet-derived growth factor-receptor and epidermal growth factor-receptor signaling and systemic administration of paclitaxel as therapy for human prostate cancer metastasis in bone of nude mice. Cancer Res. 64:4201-4208.

23. Wilkinson-Berka, J.L., et al. 2004. Inhibition of platelet-derived growth factor promotes pericyte loss and angiogenesis in ischemic retinopathy. Am. J. Pathol. 164:1263-1273.

24. Koyama, N., et al. 1994. Migratory and proliferative effect of platelet-derived growth factor in rabbit retinal endothelial cells: evidence of an autocrine pathway of platelet-derived growth factor. J. Cell. Physiol. 158:1-6.

25. Marx, M., Perlmutter, R.A., and Madri, J.A. 1994 Modulation of platelet-derived growth factor receptor expression in microvascular endothelial cells during in vitro angiogenesis. J. Clin. Invest. 93:131-139.

26. Smits, A., et al. 1989. Rat brain capillary endothelial cells express functional PDGF B-type receptors. Growth Factors. 2:1-8.

27. Battegay, E.J., Rupp, J., Iruela Arispe, L., Sage, E.H., and Pech, M. 1994. PDGF-BB modulates endothelial proliferation and angiogenesis in vitro via PDGF beta-receptors. J. Cell Biol. 125:917-928.
28. Thommen, R., et al. 1997. PDGF-BB increases endothelial migration on cord movements during angiogenesis in vitro. J. Cell. Biochem. 64:403-413.

29. Beitz, J.G., Kim, I.S., Calabresi, P., and Frackelton, A.R., Jr. 1991. Human microvascular endothelial cells express receptors for platelet- derived growth factor. Proc. Natl. Acad. Sci. U. S. A. 88:2021-2025.

30. Plate, K.H., Breier, G., Farrell, C.L., and Risau, W. 1992. Platelet-derived growth factor receptor-beta is induced during tumor development and upregulated during tumor progression in endothelial cells in human gliomas. Lab. Invest. 67:529-534.

31. Bar, R.S., et al. 1989. The effects of platelet-derived growth factor in cultured microvessel endothelial cells. Endocrinology. 124:1841-1848.

32. Lindahl, P., et al. 1998. Paracrine PDGF-B/ PDGF-Rbeta signaling controls mesangial cell development in kidney glomeruli. Development. 125:3313-3322.

33. Lindner, V., and Reidy, M.A. 1995. Platelet-derived growth factor ligand and receptor expression by large vessel endothelium in vivo. Am. J. Pathol. 146:1488-1497.

34. Edelberg, J.M., et al. 1998. PDGF mediates cardiac microvascular communication. J. Clin. Invest. 102:837-843.

35. Cao, R., et al. 2002. Angiogenesis stimulated by PDGF-CC, a novel member in the PDGF family, involves activation of PDGFR-alphaalpha and -alphabeta receptors. FASEB J. 16:1575-1583.

36. Oikawa, T., Onozawa, C., Sakaguchi, M., Morita, I., and Murota, S. 1994. Three isoforms of platelet-derived growth factors all have the capability to induce angiogenesis in vivo. Biol. Pharm. Bull. 17:1686-1688.

37. Risau, W., et al. 1992. Platelet-derived growth factor is angiogenic in vivo. Growth Factors. 7:261-266.

38. De Marchis, F., et al. 2002. Platelet-derived growth factor inhibits basic fibroblast growth factor angiogenic properties in vitro and in vivo through its alpha receptor. Blood. 99:2045-2053.

39. Uutela, M., et al. 2001. Chromosomal location, exon structure, and vascular expression patterns of the human PDGFC and PDGFD genes. Circulation. 103:2242-2247.

40. Li, H., Fredriksson, L., Li, X., and Eriksson, U. 2003. PDGF-D is a potent transforming and angiogenic growth factor. Oncogene. 22:1501-1510.

41. Luttun, A., et al. 2002. Revascularization of ischemic tissues by PlGF treatment, and inhibition of tumor angiogenesis, arthritis and atherosclerosis by anti-Flt1. Nat. Med. 8:831-840.

42. Palumbo, R., et al. 2002. Different effects of high 
and low shear stress on platelet-derived growth factor isoform release by endothelial cells: consequences for smooth muscle cell migration. Arterioscler. Thromb. Vasc. Biol. 22:405-411.

43. Rafii, S. 2000. Circulating endothelial precursors: mystery, reality, and promise. J. Clin. Invest. 105:17-19.

44. Miraglia, S., et al. 1997. A novel five-transmembrane hematopoietic stem cell antigen: isolation, characterization, and molecular cloning. Blood. 90:5013-5021.

45. Ekman, S., Thuresson, E.R., Heldin, C.H., and Ronnstrand, L. 1999. Increased mitogenicity of an alphabeta heterodimeric PDGF receptor complex correlates with lack of RasGAP binding. Oncogene. 18:2481-2488.

46. Blacher, S., et al. 2001. Improved quantification of angiogenesis in the rat aortic ring assay.
Angiogenesis. 4:133-142.

47. Ponten, A., et al. 2003. Transgenic overexpression of platelet-derived growth factor-C in the mouse heart induces cardiac fibrosis, hypertrophy, and dilated cardiomyopathy. Am. J. Pathol. 163:673-682.

48. Apte, S.M., Fan, D., Killion, J.J., and Fidler, I.J. 2004. Targeting the platelet-derived growth factor receptor in antivascular therapy for human ovarian carcinoma. Clin. Cancer Res. 10:897-908.

49. Betsholtz, C., Karlsson, L., and Lindahl, P. 2001. Developmental roles of platelet-derived growth factors. Bioessays. 23:494-507.

50. Ding, H., et al. 2004. A specific requirement for PDGF-C in palate formation and PDGFR-alpha signaling. Nat. Genet. 36:1111-1116.

51. Buschmann, I., Heil, M., Jost, M., and Schaper, W. 2003. Influence of inflammatory cytokines on arteriogenesis. Microcirculation. 10:371-379.
52. Hudkins, K.L., et al. 2004. Exogenous PDGF-D is a potent mesangial cell mitogen and causes a severe mesangial proliferative glomerulopathy. J. Am. Soc. Nephrol. 15:286-298.

53. Schaeren-Wiemers, N., and Gerfin-Moser, A. 1993. A single protocol to detect transcripts of various types and expression levels in neural tissue and cultured cells: in situ hybridization using digoxigenin-labelled cRNA probes. Histochemistry. 100:431-440.

54. Eriksson, A., Siegbahn, A., Westermark, B., Heldin, C.H., and Claesson-Welsh, L. 1992. PDGF alphaand beta-receptors activate unique and common signal transduction pathways. EMBOJ. 11:543-550.

55. Dimmeler, S., et al. 2001. HMG-CoA reductase inhibitors (statins) increase endothelial progenitor cells via the PI 3-kinase/Akt pathway. J. Clin. Invest. 108:391-397. doi:10.1172/JCI200113152. 